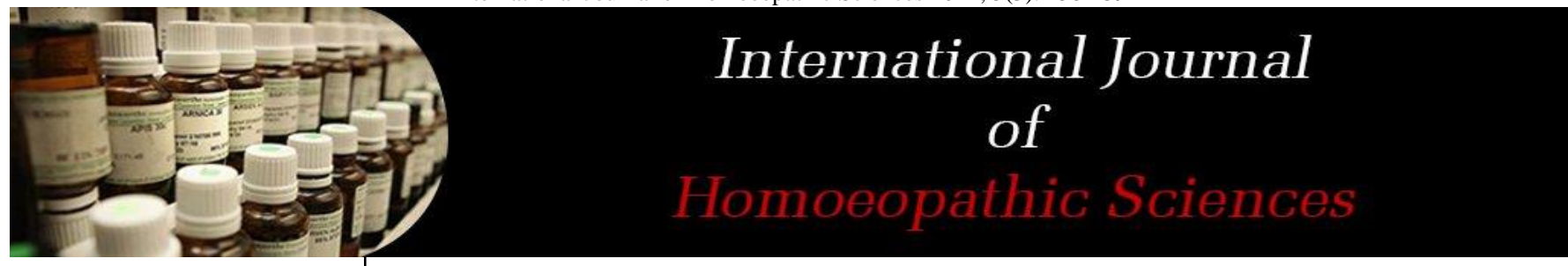

E-ISSN: $2616-4493$ P-ISSN: 2616-4485 www.homoeopathicjournal.com IJHS 2021; 5(3): 158-159 Received: 19-05-2021 Accepted: 21-06-2021

Dr. Bed Prakash Gond BHMS (NIH), MD(HOM) Lecturer, Department of Community Medicine, Netai Charan Chakravarty Homoeopathic Medical College \& Hospital, (WBUHS) Howrah, West Bengal, India
Corresponding Author: Dr. Bed Prakash Gond BHMS (NIH), MD(HOM) Lecturer, Department of Community Medicine, Netai Charan Chakravarty Homoeopathic Medical College \& Hospital, (WBUHS) Howrah, West Bengal, India

\section{Loss of smell (Anosmia/Hyposmia) due to SARS-CoV2 infection \& Its Homoeopathic Indications}

\section{Dr. Bed Prakash Gond}

DOI: $\underline{\text { https://doi.org/10.33545/26164485.2021.v5.i3c.420 }}$

Abstract

There has been some renewed interest in current situations in disorders of olfaction. Decreased sense of smell can lead to significant impairment of quality of life, including taste disturbance and loss of pleasure from eating with resulting changes in weight and difficulty in avoiding health risks such as spoiled food or leaking natural gas. When validated smell identification or threshold tests are used, its reveal quite a high prevalence of hyposmia and anosmia in certain groups, especially the age group between 20 to 40 years old. As Homoeopaths, we are most likely to see the symptoms similarities in patients with chronic rhinosinusitis, and this now appears to be due more to SARS CoV2 Infection.

Keywords: SARS CoV2, COVID 19, anosmia, Hyposmia, olfactory disfunction, chronic miasm, sensorium; syphilis, sycotic, syco-syphilitic

\section{Introduction}

Sense of smell is well-developed in lower animals to give warning of the surrounding dangers but it is comparatively less important in man. Still it is important for pleasure and for enjoying the essence of food. When nose is blocked, food tastes bland and unpalatable. Anosmia is a total loss of smell while Hyposmia is partial loss of smell. They can result from stimulation of fibres of the trigeminal nerve and causes irritation in the nose which results in nasal obstruction due to nasal polyp, enlarged turbinates or oedema of mucus membrane as in common cold, allergic or vasomotor rhinitis.

\section{In relations with SARS-CoV2}

Anosmia or Hyposmia is the main neurological symptom and one of the earliest and most commonly reported indicators of COVID-19. It suggests better predicts the disease than other well-known symptoms such as fever and cough, but underlying mechanisms for loss of smell in patients with COVID-19 is still unknown.

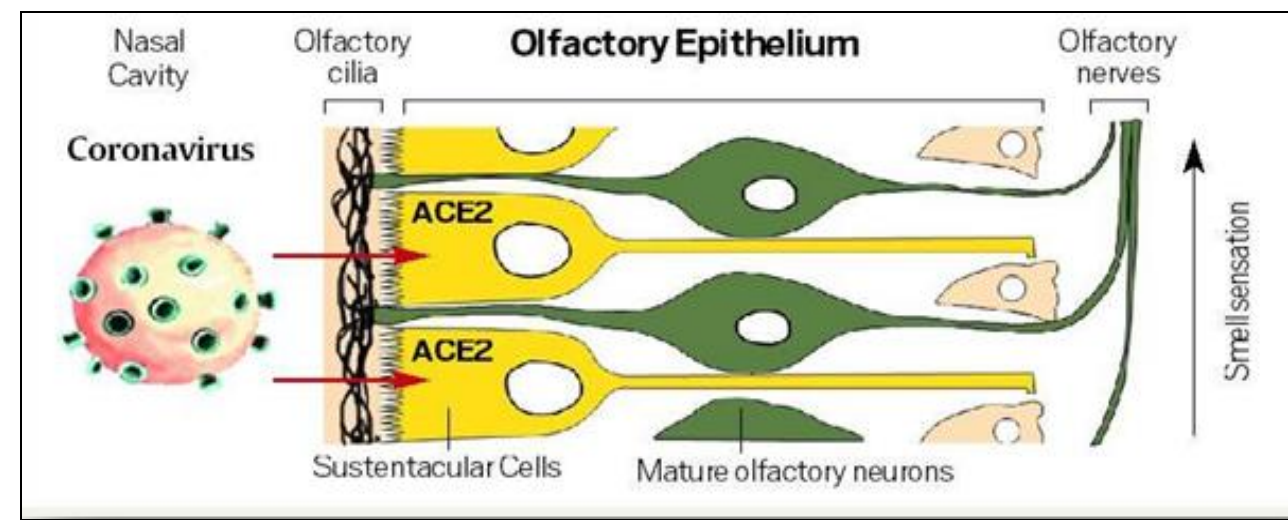

The virus, the protein and the Mechanism of smell

Recently, it is found that the olfactory cell types in the upper nasal cavity most vulnerable to infection by SARS-CoV2, the virus that causes COVID-19. Surprisingly, sensory neurons that detect and transmit the sense of smell to the brain are not among the vulnerable cells types. The olfactory sensory neurons do not express the gene that encodes the ACE2 receptor protein, which SAR-CoV2 uses to enter human cells. Instead, ACE2 is expressed in cells that provide metabolic and structural support to olfactory sensory neurons, as well as certain 
populations of stem cells and blood vessels cells. The findings suggest that infection of non-neural cell types may be responsible for anosmia in COVID-19 patients and help inform efforts to better understand the progression of the disease. Hence, the novel corona virus changes the sense of smell in patient not by directly infecting neurons but by affecting the function of supporting cells. This implies that in most cases, SARS-CoV2 infection is unlikely to permanently damage olfactory neural circuits and lead to persistent anosmia.

The COVID-19 patients typically recover their sense of smell over the course of weeks, much faster than the months. It can take to recover from anosmia caused by a subset of viral infections known to directly damage olfactory sensory neurons.

In addition, many viruses cause temporary loss of smell by triggering upper respiratory tract issues such as stuffy nose with severe headache. Some COVID-19 patients, however, experience anosmia without any nasal congestion. Anosmia seems like a curious phenomenon, but it can be devastating for the small fraction of people in whom it's persistent. It can have serious psychological consequences and could be a major public health problem if we have a growing population with permanent loss of smell.

\section{How Homoeopathy Can Helps to Cure Anosmia / Hyposmia:}

Homoeopathy is one of the most popular holistic systems of medicine. The selection of remedy is based upon the theory of individualization and symptoms similarity by using holistic approach. The aim of Homoeopathy is not only to treat Anosmia/Hyposmia symptoms but to remove its underlying cause and individual susceptibility.

\section{Miasmatic Approach}

Dr. J.H. Allen in his book "Chronic Miasm" in chapter "Sensorium" stated that sometimes the smell may be diminished or lost but this comes more frequently in syphilis or sycosis, these have complete loss of smell and taste, also of hearing. A thickening of the membrane or enlargement of the turbinated bodies are always due congestion. A red nose with enlarged capillaries depends on a sycotic element or over stimulation of the organism. Syphilis produces ulceration, large thick crust, known as clinkers, often filling the whole nasal cavity; frequently they have to be removed, but soon form again. Basically, the law similaris is employing the destructive elements therapeutically in curing the destructive syco-syphilitic miasm.

\section{Susceptibility and Cure}

Cure is nothing but the result of satisfying the morbid or altered susceptibility. Dr. J.T. Kent says "When susceptibility is satisfied, there is cessation of cause, and when cause ceases to flow in to ultimate, not only do ultimate cease but case itself has already ceased". As hunger demands food, so disease demands medicine. But the demand is always consistent with the universal law. It is for the symptomatically similar medicine, because that is the only thing that really satisfies the susceptibility. Dr. H.A. Robert says "The similar remedy or the similar disease satisfies susceptibility and establishes immunity". When a Homoeopathically selected remedy is administered to a sick person, the disappearance of symptoms indicates that the person is susceptible to the curative remedy and the powerful similar remedy is capable enough to replace the weaker miasmatic disease. Dr. Stuart Close says "The kind and the degree of reaction to medicines depend upon the degree of susceptibility of the patient". Hence, cure is brought by simply satisfying the morbid susceptibility of the patient by the similar remedies.

\section{Homoeopathic Therapeutics}

Belladonna: Nocturnal shootings in the nose; Swelling, redness and burning it the point of the nose; Inflammatory swelling and redness of the external and internal nose; Great dryness of the nose; Sense of smell either too sensitive, especially to tobacco smoke or diminished.

Calcarea Carbonica: Inflammation of the nose, with redness and swelling; Sometimes producing fainting; Fetid smell from the nose; Sense of smell dull or exceedingly sensitive; Painful dryness in the nose; fetid odour before the nose, as if from a dunghill, rotten eggs, or gunpowder.

Kali Iodatum: Tearing of upper part of nostrils; Burning in nostrils; in upper part, with feeling as if a leaf were in front of it; Stuffiness and dryness of nose; Stoppage (in morning), with running of clear water; corrosive and burning; Redness and swelling of nose; loss of smell.

Mercurius: Swelling of the bones of the nose (external nose, as bridge of the nose, may swell up very large on both sides), with painful sensitiveness to touch; Tension, pressure, and sensation of heaviness in nose; Inflammatory swelling and shining redness of nose; obstruction and dryness of nose; Putrid smell from nose.

Plumbum Metallicum: Erysipelatous inflammation of nose; Red, purulent vesicles in nasal angles; Fetid smell in nose; Loss of smell; Obstruction of nose; Accumulation of tenacious mucus in nostrils, which can only be expelled by way of the nasal fossae.

\section{References}

1. Dhingra PL, Diseases of Ear, Nose and Throat, Read Elsevier India Private Limited Publication, Fourth Edition.

2. Allen JH, The Chronic Miasm, Bjain Publishers (P) LTD., reprint 2004.

3. Clarke JH, A Dictionary of Practical Materia Medica.

4. Boericke W, Pocket Manual of Homoeopathic Materia Medica and Repertory.

5. Narendra Babu G, Comprehensive Study of Organon, Bjain Publishers (P) LTD.

6. Sandeep Robert Datta, associate professor, neurobiology in the Blavatnik Institute at HMS. https://hms.harvard.edu 